\title{
"Reading Is the Key to Knowledge". Las Lecturas Guiadas como Instrumento de Aprendizaje en las Asignaturas de Historia Económica en la Facultad de Ciencias del Trabajo
}

RESUMEN:

\author{
Isabel Bartolomé Rodríguez* \\ Francisco Bernal Garcia * \\ Alicia Gil Lázaro* \\ Pablo Gutiérrez González* \\ Marcial Sánchez Mosquera*
}

El propósito de este artículo es presentar, al tiempo que evaluar, la experiencia que durante el curso 2011-2012 se desarrolló por parte de los profesores del Área de Historia e Instituciones Económicas con el uso de guias de lectura en el marco de las clases prácticas en la Facultad de Ciencias del Trabajo de la Universidad de Sevilla. Se perseguía desarrollar una estrategia didáctico-metodológica donde el alumno sea un elemento activo en el proceso de enseñanza aprendizaje, procurando dar los primeros pasos en su "alfabetización académica". Las valoraciones de los estudiantes, al final del cuatrimestre, nos animan a perseverar en el uso de estas guías.

Palabras clave:

Didáctica; Relaciones Laborales; Alfabetización Académica; Clases Prácticas.

ABSTRACT:

This article focuses on an experience developed during the academic year 2011-2012 with the use of reading-guides in the framework of the practical sessions in the Faculty of Work Science of the University of Seville. This experience has been aimed by the faculty of the History and Economic Institutions' Area. The reading-guides were conceived as

* Área de Historia e Instituciones Económicas. Universidad de Sevilla • mbartolome@us.es 
an strategy in order to promote the active role of the student in the teaching-learning process. The reading-guides are considered as the first step in an "academic literacy" process. The assessments of students, at the end of the semester, encourage us to persevere in the use of these guides.

KEYWORDS:

Portfolios; student evaluation; learned controlled; teaching methods; class organization; higher education; competence; resource teacher.

\section{INTRODUCCIÓN}

El propósito de este artículo es dar a conocer y evaluar un instrumento de aprendizaje, las "Guías de Lectura", empleado en las sesiones prácticas de las asignaturas de Historia de las Relaciones Laborales e Historia Económica del Trabajo en la Facultad de Ciencias del Trabajo de la Universidad de Sevilla. La guía se concibe como herramienta de intermediación crítica entre el lector y el texto académico, efectuando sucesivamente las preguntas que el texto va contestando, para fijar la atención en los contenidos principales de la lectura y aclarar la estructura del texto. Esta herramienta está destinada a la lectura individual, pero se complementa con una clase práctica. Durante la misma, a) el profesor enfatiza la estructura y los contenidos principales del texto; b) los alumnos comparten en un trabajo de grupos/pares los conceptos básicos del texto; c) se propicia la participación y el debate en plenaria; por último, se realiza una prueba escrita individual sobre los contenidos básicos del texto. Estas últimas actividades potencian otras competencias y evalúan otras habilidades, complementando el ejercicio de lectura comprensiva. Aquí, la experiencia en el uso de estas guías durante el curso 2011-2012 se ha sometido al juicio de los propios estudiantes: 222 alumnos de ambas asignaturas en la Facultad de Ciencias del Trabajo de esta Universidad han respondido a cuestionarios para su valoración.

La comunicación consta de tres partes. En la primera, se justifica el papel de la lectura de textos académicos en el currículum de los alumnos universitarios, constatando las dificultades y las ventajas que su uso conlleva en los primeros cursos. En la segunda, se desgrana el cometido de las prácticas en las asignaturas de Historia de las Relaciones Laborales en el proceso de aprendizaje propuesto a raíz del Libro Blanco para la puesta en práctica del nuevo Grado en Relaciones Laborales y Recursos Humanos. Ahí mismo, se explica cómo surgió y cómo se hace uso efectivo de esta propuesta de intermediación didáctica. La tercera parte de la comunicación evalúa los resultados de las encuestas contestadas por los alumnos de estas asignaturas durante el curso académico 2011-2012, a fin de valorar su grado de conformidad con el uso de diferentes soportes --textos, vídeos, etcétera...-- y con el uso concreto de 
las Guías de Lectura en las prácticas de estas asignaturas. Finalmente, proponemos unas conclusiones.

\section{1.- $\quad$ LA LECTURA DE TEXTOS ACADÉMICOS EN LA ENSEÑANZA UNVIERSITARIA: DE LA FRUSTRACIÓN AL APRENDIZAJE}

La puesta en marcha del Espacio Europeo de Educación Superior (EEES) ha traído consigo nuevas formas de entender el proceso de aprendizaje de los estudiantes universitarios. La asistencia a clases teóricas y la realización de exámenes finales han dejado de ser los únicos parámetros a través de los cuales es evaluado el trabajo del estudiante. Junto a ellas, se han abierto paso toda una serie de "actividades complementarias", de carácter eminentemente práctico, que buscan incentivar al estudiante a adoptar una actitud más proactiva a la hora de construir el conocimiento y facilitarle la adquisición de habilidades esenciales para el dominio de las diferentes disciplinas académicas. Una de las "actividades complementarias" más utilizadas es la lectura de textos académicos. Los estudiantes deben leer textos relevantes para la asignatura, previamente seleccionados por el profesor, y realizar algún tipo de prueba mediante la cual acreditan la lectura y la comprensión de los mismos.

La realización de pruebas basadas en la lectura de textos académicos parece, en principio, una actividad poco compleja, de fácil organización y plenamente adaptada a las habilidades y competencias que se esperan de un estudiante universitario. Sin embargo, la realidad nos demuestra que los resultados de este tipo de pruebas suelen ser poco alentadores, siendo muy alto el porcentaje de alumnos que no logran completarlas de manera satisfactoria debido, fundamentalmente, a dificultades a la hora de interpretar correctamente los textos académicos que les sirven de base. Las insuficientes habilidades lectoras de los estudiantes universitarios han terminado por convertirse en motivo recurrente de queja por parte de los profesores. "Los estudiantes no comprenden lo que leen", "los estudiantes no prestan atención a lo que leen", "los estudiantes no saben leer"... Son expresiones que escuchamos frecuentemente por parte de profesores universitarios que intentan enseñar a través de la lectura de textos académicos.

Lejos de lo que, a priori, podríamos pensar, éste no es un problema exclusivo del sistema universitario español. Muy al contrario, ha sido constatado en numerosos países, tanto desarrollados como en vías de desarrollo. En Estados Unidos, Australia o Argentina las insuficientes habilidades lectoras de los estudiantes universitarios han llegado a ser, incluso, tema de debate en los medios de comunicación de masas (Flores y Natale, 2004). La explicación que los profesores universitarios dan a este fenómeno suele ser muy parecida en todos estos países: los estudiantes no superan satisfactoriamente las pruebas basadas en la lectura de textos académicos, porque acceden a la universidad con una mala preparación, incubada durante los años em- 
pleados en completar estudios de educación secundaria. La educación secundaria debería proporcionar a los estudiantes las habilidades necesarias para afrontar con garantías la lectura de textos académicos pero, en la práctica, no lo está haciendo, por lo que se impone una revisión en profundidad de la misma (Carlino, 2003).

El profesor universitario que constata que sus estudiantes carecen de las habilidades necesarias para afrontar con garantías la lectura de textos académicos suele reaccionar a este hecho con altas dosis de frustración. El profesor ha organizado "actividades complementarias" basadas en la lectura de textos académicos dando por hecho que sus estudiantes los leerán con fluidez y que podrá centrar su trabajo de clase en la explicación de las ideas, conceptos y tesis expresados en los mismos. Pero el profesor descubre que este método de trabajo resulta inviable: los estudiantes no comprenden sus explicaciones, porque carecen de las habilidades necesarias para trabajar adecuadamente con los textos que les sirven de base. Por otra parte, el profesor no cree que enseñar a los estudiantes cómo leer textos académicos entre dentro de sus funciones, dado que considera que este aprendizaje debería haber sido completado por los estudiantes durante sus estudios secundarios. El resultado suele ser el fracaso de la propuesta docente elaborada por el profesor.

Desde hace aproximadamente una década, los problemas de comprensión lectora de los estudiantes universitarios están siendo estudiados por diferentes especialistas en didáctica, especialmente en los ámbitos anglosajón y latinoamericano (Geisler, 1994; Johns, 1997; Narvaja de Arnoux, Di Stefano y Pereira, 2002; Carlino, 2006; Narváez Cardona y Cadena Castilla, 2008). Estos autores enfocan los problemas de lectura de los estudiantes universitarios desde una perspectiva muy diferente a la habitualmente adoptada por los profesores. Parten de la idea de que los estudiantes sí han aprendido a leer durante sus estudios secundarios. El problema radica en que las habilidades lectoras que han desarrollado durante la educación secundaria no son exactamente las que se espera de ellos en la educación universitaria. La cultura lectora que impera en la educación secundaria es muy diferente a la que se exige en la educación universitaria.

En la educación secundaria los textos son concebidos como fuente indiscutible de conocimiento. Se parte de la idea de que los textos contienen todo lo que resulta necesario para superar satisfactoriamente la asignatura. Se trabaja con textos que omiten los métodos a través de los cuales se ha construido el conocimiento y la controversia epistemológica que dicho proceso de construcción del conocimiento haya podido originar. Son textos que abordan el conocimiento como algo ahistórico, único y exclusivo (Carlino, 2006: 90-91). Por el contrario, en la educación universitaria se espera que el estudiante sea capaz de desarrollar una lectura crítica del texto. Que identifique el proceso dialéctico que ha llevado a la construcción del conocimiento -los posicionamientos de las distintas escuelas y autores. El estudiante debe ser 
consciente de que no siempre toda la información que necesita se encuentra en el texto sino que, a menudo, ésta se obtiene relacionando el contenido del texto con otras informaciones -explicaciones de clase u otros textos.

El estudiante que accede por primera vez a estudios universitarios desconoce el aparato crítico empleado en los textos académicos -notas a pie de página, citas, tablas, gráficos- y la función que desempeña dentro de los mismos. Se enfrenta a graves dificultades a la hora de jerarquizar las ideas e informaciones expuestas en el texto, siendo habitual que no sepa discernir la diferente importancia que cabe atribuir a una tesis fundamental y a un ejemplo. Tiende a desarrollar una lectura ahistórica del texto, no siendo capaz de ubicar la información que le proporciona el texto en un determinado contexto histórico y de identificar las secuencias temporales que jalonan el desarrollo de los acontecimientos, las ideas y los conceptos. Ignora, en fin, la dimensión dialéctica y crítica de la lectura de un texto académico, tendiendo a considerarlo como una fuente de autoridad unívoca. Para él, el texto es la fuente en la que va a encontrar la interpretación "oficial" de los hechos, que le permitirá aprobar la asignatura. No concibe que un texto académico pueda ser sometido a crítica o, incluso, puesto en cuestión. No entiende que un mismo acontecimiento pueda ser interpretado de diferente forma por un autor liberal, keynesiano o marxista.

El estudiante que accede a estudios universitarios debe enfrentarse a una nueva cultura académica. Ello implica ser capaz de desarrollar nuevas prácticas intelectuales. Entre éstas, ocupa un lugar preeminente la capacidad de leer textos académicos. El paso de la cultura académica característica de la educación secundaria a la nueva cultura académica característica de la educación superior es considerado por numerosos autores como un proceso de alfabetización académica (Carlino, 2006:13).

El concepto de alfabetización académica parte de la idea de que es habitual que, a lo largo de su vida, un individuo se vea obligado a completar diferentes "alfabetizaciones". Las habilidades de leer y escribir no son algo que éste adquiera una única vez, capacitándole para la lectura y la escritura de todo tipo de textos. Muy al contrario, cada vez que alguien desea introducirse en un ámbito cultural nuevo, debe aprender a leer y escribir "de nuevo", para adquirir las claves de lectura y escritura específicas de dicho ámbito cultural. El estudiante que inicia estudios universitarios se enfrenta a un nuevo ámbito cultural -a una nueva cultura académica. La plena integración en esa nueva cultura implica, necesariamente, la adopción de una nueva cultura lectora -una nueva forma de leer- $\mathrm{y}$, para ello, es necesario que el estudiante complete un proceso de alfabetización académica.

El profesor universitario tiende a pensar que la cultura lectora que el estudiante ha adquirido durante la educación secundaria le habilita automáticamente para ser un buen lector de textos académicos. Sin embargo, como hemos visto, las culturas lectoras de la educación secundaria y de la educación universitaria son cualitativamente 
distintas. En último extremo, el profesor piensa que los estudiantes desarrollarán las nuevas habilidades lectoras requeridas por la universidad de una manera espontánea y que lograrán hacerlo en un breve período de tiempo.

Los especialistas en didáctica que han desarrollado el concepto de alfabetización académica contemplan el problema desde una perspectiva distinta. Consideran que el estudiante necesita ser formado en las nuevas habilidades lectoras requeridas por el nivel universitario. El estudiante necesita que se le informe explícitamente acerca de qué tipo de lectura se está requiriendo de él y, al mismo tiempo, que se le proporcionen los instrumentos prácticos para llevar a cabo ese tipo de lectura.

Numerosas universidades anglosajonas y latinoamericanas han apostado decididamente por esta vía, ofreciendo a sus estudiantes cursos introductorios de lectura y escritura académicas. Son cursos que se desarrollan durante el primer año de universidad y que van encaminados a dotar a los estudiantes de las habilidades lectoescritoras necesarias para enfrentarse exitosamente a los requerimientos que el acceso a una nueva cultura académica les impone. Sin embargo, entre los autores que han profundizado en el concepto de alfabetización académica predomina la idea de que tales cursos introductorios no cumplen con sus objetivos debido a que se imparten desgajados del contenido específico de las distintas asignaturas que los estudiantes han de cursar durante sus estudios universitarios (Carlino, 2006:21). No tiene sentido concebir la lectura y la escritura académicas como habilidades desvinculadas de las distintas disciplinas académicas que los estudiantes han de cursar a lo largo de sus estudios universitarios, dado que cada disciplina genera sus propias formas específicas de lectura y escritura (Hyland, 2000); Cassany, 2006:21). La adquisición de las habilidades lectoras adecuadas a las nuevas disciplinas académicas se debe producir, por lo tanto, dentro del contexto de las mismas. Ha de ser el profesor de la asignatura el que, paralelamente a la explicación de los textos académicos propios de la asignatura, oriente al estudiante acerca de cómo leerlos. El profesor ha de ser un "guía" que introduzca al estudiante en las nuevas habilidades esenciales para formar parte de la comunidad académica en la que éste, a través de sus estudios universitarios, pretende integrarse (Carlino, 2003b); Estienne, 2004).

Los especialistas en didáctica que propugnan el paradigma de la alfabetización académica proponen a los profesores diferentes instrumentos destinados a introducir a los estudiantes en las técnicas de lectura características de su disciplina. Entre tales instrumentos adquiere una relevancia particular el uso de guías de lectura, entendidas como documentos de trabajo que, de una manera dinámica -a menudo a través de cuestionarios que obligan al estudiante a indagar críticamente en la estructura y contenido del texto-, se convierten en un punto de apoyo que sirve al estudiante para iniciarse en las nuevas habilidades requeridas por la lectura de textos académicos. 
La alfabetización académica se convierte, de este modo, en un complemento imprescindible a la formación teórica en los contenidos de cada asignatura. Es la faceta del aprendizaje que permite al estudiante adquirir las habilidades e instrumentos esenciales para el dominio de su disciplina e integrarse, como miembro de pleno derecho, en la comunidad académica de la que aspira a formar parte. La alfabetización académica no pretende, de ningún modo, compensar los fallos e insuficiencias de la educación secundaria, sino que busca ser una vía para introducir al estudiante en los nuevos usos y prácticas intelectuales que se requieren de él en la educación universitaria. En este sentido, resulta significativo que en aquellos países donde las instituciones universitarias promueven la incorporación de la alfabetización académica a los contenidos docentes de las asignaturas, los estudiantes la valoren de una manera altamente positiva, considerándola, a menudo, como uno de los aspectos de su formación que más les ayuda a superar los retos a los que deben hacer frente en el día a día de su actividad universitaria (Carlino, 2003).

\section{2.- $\quad$ LA LECTURA, INSTRUMENTO DE APRENDIZAJE PARA LAS ASIGNATURAS DE HISTORIA EN EL GRADO DE RELACIONES LABORALES Y RECURSOS HUMANOS}

A partir del curso académico 2009/2010 se implantó de manera oficial en la Facultad de Ciencias del Trabajo de la Universidad de Sevilla el nuevo Grado en Relaciones Laborales y Recursos Humanos. Este Grado incorporó en su Plan de Estudios dos asignaturas de Historia: "Historia de las Relaciones Laborales" (dentro de la categoría de Formación Básica del primer curso académico) e "Historia Económica del Trabajo" (como Optativa del segundo curso académico). ${ }^{1}$ Ambas forman parte del bloque temático de "Historia social y de las relaciones laborales", el cual se engloba, junto a otros diez (cada uno con una carga distinta de créditos ECTS), en el proyecto de Grado presentado ante la Agencia de Evaluación de la Calidad y la Acreditación (ANECA) bajo la denominación de Libro Blanco. Titulo de Grado en Ciencias Laborales y Recursos Humanos, que fue aprobado oficialmente en 2005 por esta entidad evaluadora y que ha constituido el modelo seguido por las universidades españolas que han implantado el Grado en Relaciones Laborales y Recursos Humanos hasta ahora ( Lerma Montero, 2005)2.

1. La primera de ellas es una asignatura nueva y la segunda ya formó parte del antiguo Plan de Estudios de la Licenciatura de Ciencias del Trabajo (2002).

2. En dos ponencias presentadas en los Encuentros de Didáctica de la Historia Económica posteriores a 2005 se analiza con cierto detalle la progresiva implantación del Grado en las universidades españolas, las diversas denominaciones que ha recibido así como las diferentes asignaturas de Historia que se han incluido en sus respectivos Planes de Estudios. Véase Galán García y Manzanares Martínez (2010) y González Arce y otros (2008:273-288). Agustín Galán amplía posteriormente el debate sobre el proceso de convergencia desde el Grado de Relaciones Laborales en términos nacionales. Galán García (2011:15-45). 
Tal y como afirman Galán García y Herraiz Martín (2005:17-31), la asignatura de Historia ha formado parte de casi todos los planes de estudios españoles desde 1929 hasta la actualidad, tanto en las antiguas Escuelas de Graduados Sociales, como posteriormente en las Escuelas Universitarias y, de manera más reciente, en las Facultades de Ciencias del Trabajo, impartida en la mayoría de las universidades por el Área de conocimiento de Historia e Instituciones Económicas. Así ha sido al menos en lo que respecta a la Universidad de Sevilla, donde dicha Área no sólo ha aportado ininterrumpidamente su especialización y experiencia docente en las distintas asignaturas — desde las extintas "Historia Social y Política Contemporánea" e "Historia Económica de las Relaciones Laborales" a las actuales ya citadas - sino que ha sido, además, un foco académico de importancia en el panorama historiográfico nacional, dentro de la investigación en la historia económica del trabajo, con un fuerte impulso especialmente a lo largo de las décadas de 1990 y $2000^{3}$.

En el Libro Blanco... se especifican los objetivos que persigue el nuevo Grado así como los contenidos formativos mínimos, capacidades, destrezas y habilidades del bloque temático de "Historia social y de las relaciones laborales". Destacamos, entre estas últimas, la capacidad para la contextualización, comparación y análisis crítico "de los fenómenos, sucesos y procesos que tienen lugar en la evolución histórica del trabajo, las relaciones laborales y los mercados de trabajo", así como las destrezas para el análisis de "realidades sociales complejas pasadas y presentes" y el dominio de procedimientos, instrumentos y terminología de la historia para el análisis socio-laboral, en tanto dichas bases programáticas han constituido nuestro punto de partida para la construcción de una herramienta de trabajo desde la planificación de la enseñanza y las estrategias didácticas (Lerma Montero, 2005:256).

En efecto, a través de esta herramienta que aquí presentamos, se ha pretendido aplicar una estrategia didáctico-metodológica donde el alumno sea un elemento activo en el proceso de enseñanza aprendizaje y crear, ante todo, las condiciones adecuadas para su aprendizaje autónomo y continuo, objetivos prioritarios del nuevo Grado (Lerma Montero, 2005:241). Asimismo, la planificación docente ha llevado a desarrollar diferentes técnicas de trabajo cooperativo y de dinámica de grupos en el aula. Para ello se ha enfatizado el carácter procedimental de las denominadas "actividades complementarias", es decir, los contenidos prácticos que los alumnos deben obligatoriamente realizar en cada materia, atendiendo a los requerimientos que el Espacio Europeo de Educación Superior (EEES) prevé como complemento a las lecciones magistrales tradicionales del profesor: "aquellos contenidos [...] que si bien requieren de un conocimiento teórico previo de la

3. Las dos publicaciones más significativas de este esfuerzo investigador emanaron de dos encuentros nacionales celebrados en Sevilla en 1997 y 2000. Arenas Posadas, Florencio Puntas y Martínez Ruiz (1998) y Arenas Posadas, Florencio Puntas y Pons Pons (2001). Véase también Bernal, Collantes de Terán y García Baquero (2008). 
materia por parte del alumno [...] consisten en todo tipo de ejercicios y actividades con los que el alumno pondrá en práctica tales conocimientos teóricos previos y desarrollará las capacidades a lograr" (González Arce y otros, 2008:283).

\section{1.- El PAPEl De Las aCtividades COMPLEMENTARias}

Las dos asignaturas que nuestra Área imparte en el nuevo Grado de Relaciones Laborales y Recursos Humanos tienen una carga de seis créditos ECTS cada una. Los objetivos docentes específicos y competencias en ambas se ajustan cabalmente a los lineamientos planteados en el Libro Blanco de modo que dedican 37,5 horas presenciales en el aula (y 60 horas no presenciales) al desarrollo de los contenidos teóricos y 22,5 horas a las actividades prácticas (30 horas no presenciales) (González Arce y otros, 2008:283). Las competencias transversales que se pretende conseguir presentan, en los dos casos, el siguiente orden de prioridad: "capacidad de análisis y síntesis, conocimientos generales básicos, habilidades para recuperar y analizar información desde diferentes fuentes, capacidad de crítica y autocrítica, reconocimiento a la diversidad y multiculturalidad, capacidad de aprender, comprensión de culturas y costumbres de otros países". ${ }^{4}$

A lo largo del presente curso académico 2011/2012, la apuesta del equipo de profesores al frente de estas dos asignaturas se ha centrado en el reforzamiento de la lectura comprensiva, y para ello ha llevado a cabo una selección de textos, y otros soportes, con el fin de cumplimentar debidamente las competencias transversales antes citadas en lo referente a la planeación docente de las actividades prácticas. ${ }^{5}$ Se han adoptado hasta ahora tres formatos:

Lecturas: artículos de investigación y pequeños fragmentos de manuales (en torno a las quince-veinte páginas cada lectura).

Proyección de videos: documentales y fragmentos de películas (en torno a los cuarenta y cinco minutos de duración cada proyección).

Charlas-taller en el aula a cargo de especialistas invitados (la actividad ocupa el tiempo de una clase completa, alrededor de una hora y cuarenta y cinco minutos).

Por otro lado, se ha tratado de combinar los tres formatos en función de los temas abordados y los materiales disponibles para ello, de forma tal que las activida-

4. Programa de la asignatura "Historia de las Relaciones Laborales". Disponible en: http://www.us.es/estudios/ grados/plan_178/asignatura_1780007; Programa de la asignatura "Historia Económica del Trabajo". Disponible en: http://www.us.es/estudios/grados/plan 178/asignatura 1780018 [Consultados el 24.IV.2012]. La primera asignatura se desarrolla en el primer cuatrimestre del año académico mientras que la segunda se ubica en el segundo cuatrimestre.

5. En la Universidad de Sevilla las clases, teóricas y prácticas, tienen dos horas de duración, dos días a la semana. 
des acompañaran y/o complementaran a los contenidos teóricos desarrollados por los profesores en el aula, y tratando, en todo caso, de que no se superaran las seis o siete pruebas en total a lo largo de un cuatrimestre. El procedimiento común a los diferentes materiales combinaba, como establece la normativa universitaria, el trabajo de los alumnos en el aula, guiado por el docente, con el estudio individual fuera del aula, a través de un instrumento de trabajo construido por el grupo de profesores para cada ejercicio, que enlaza ambos espacios de actuación (el trabajo en el aula con el estudio autónomo): las guías de lectura (o cuestionarios), elaboradas tanto para las lecturas como para las proyecciones aunque, lógicamente, cada formato haya requerido metodologías de trabajo distintas. ${ }^{6}$

En primer lugar, citaremos la metodología que más usualmente hemos utilizado. El procedimiento seguido en las prácticas a través de la lectura de un texto es el siguiente:

La guía de lectura se entrega una semana antes de que se realice la práctica en el aula.

Los alumnos leen el texto en esos días previos a la práctica y responden a las preguntas de la guía de lectura o cuestionario.

El día fijado para la actividad complementaria en el aula se destina a comentar en plenario las preguntas más importantes o difíciles; se resuelven dudas y se amplían un tanto los conocimientos, contextualizando al autor en su tiempo y en una determinada corriente historiográfica y el texto en el conjunto de la obra del autor y en relación a otros textos.

Después se divide al grupo en varios subgrupos, de manera que cada uno de ellos trabaje por separado; primero se pide que se pongan de acuerdo en las cinco o seis ideas más importantes de la lectura y, posteriormente, que realicen una síntesis breve de la misma, que debe ser leída en voz alta por un portavoz de cada grupo y valorada críticamente por el resto de compañeros. ${ }^{7}$

El profesor circula por los grupos, dialogando con ellos, preguntando, respondiendo dudas y asegurándose de que su trabajo se desarrolle con normalidad. Los grupos han de conformarse de manera rápida y lo más espontánea posible, o bien acudiendo a la lista de clase o con el orden en el ya están sentados los estudiantes, tratando de que cambien en cada práctica.

6. La planificación de cada práctica se realiza en función del ritmo del profesor en las clases teóricas, de tal modo que la práctica, insistimos, coincida aproximadamente con los contenidos a los cuales complementa o hace referencia; su fijación en el calendario ha de estar sujeta, por tanto, a una cierta flexibilidad, sabiendo, a priori, que al menos una o dos prácticas deben ser realizadas en un mismo mes (o lo que es lo mismo, una práctica cada dos o tres semanas, ocupando al menos una sesión).

7. Si los alumnos han cumplimentado debidamente la guía en sus casas, debemos suponer que estas dos tareas - la búsqueda de las cinco o seis ideas claves y la síntesis- ya está hecha con antelación. Es por ello que enfatizamos que su misión en el trabajo en grupos en el aula es ponerse de acuerdo y defender lo que cada cual ha hecho ya individualmente. 
La metodología de trabajo varía notablemente cuando se trata de prácticas a través de la proyección de videos en el aula:

La guía de lectura se entrega el mismo día de la proyección y antes de dar inicio a la misma se pide a los alumnos que lean las preguntas detenidamente.

El cuestionario se debe responder al mismo tiempo que se proyecta el documental o fragmento de película, actividad que requiere un mayor esfuerzo de concentración.

Después se comentan las preguntas, en plenario o por grupos más reducidos; se establecen analogías entre lo estudiado en las clases teóricas y el material fúlmico proyectado y se resuelven dudas, generando un debate entre los grupos siempre que se pueda, a través de enlaces comparativos (diferencias y similitudes) con el presente.

La actividad finaliza de modo similar a la anterior: los grupos deben ser capaces de seleccionar las cinco o seis ideas más importantes de la proyección y construir a partir de ellas una síntesis breve que ha de ser leída y evaluada por todos los grupos —o sólo por varios, en función del tiempo disponible - antes de la terminación del ejercicio.

Finalmente, en las charlas-taller se invita a un profesor especialista en una determinada área de la historia de las relaciones laborales y/o la historia económica del trabajo, para que imparta una conferencia en el aula frente a los alumnos y la metodología aquí es la tradicional en este tipo de actividades:

El procedimiento ideal es que el profesor envíe previamente un texto relativo al tema del que va a hablar, ya sea de su autoría o de otro autor/es.

El texto se distribuye entre los alumnos con tiempo para que lo lean en casa.

Durante la conferencia, los alumnos deben estar atentos y tomar notas, tanto de lo que dice el conferenciante como de la presentación en Power Point que utilice.

Finalizada la misma, se abre un turno de preguntas y un diálogo entre el profesor y los alumnos, de tal forma que los alumnos puedan relacionar lo que han leído en casa y lo que han escuchado en el aula.

Sobra decir que para hacer viable este último formato de práctica, los conferenciantes invitados han de ser preferentemente personas cercanas, con disponibilidad de horario, pero profesionales expertos en sus áreas. ${ }^{8}$

En cualquiera de sus tres modalidades, las actividades complementarias se evalúan a través del desempeño individual y colectivo demostrado por los alumnos en el aula (realización puntual de las guías, participación activa en los debates, papel como

8. En las dos charlas-taller impartidas en el curso 2011-2012 han participado el Dr. Agustín Galán García, Decano de la Facultad de Ciencias del Trabajo de la Universidad de Huelva, y el Dr. Carlos Arenas Posadas, ex-decano de la Facultad de Ciencias del Trabajo de la Universidad de Sevilla, abordando sendos temas de su especialidad: los accidentes de trabajo en España a lo largo del siglo XX y los mercados de trabajo de la década de 1980 a la actualidad. 
portavoces de sus grupos, interacción con los profesores invitados, etc.), de todo lo cual el profesor debe tomar buena nota en cada momento. Sin embargo, bajo este esquema que proponemos, la adquisición de nuevos conocimientos en el proceso de enseñanza aprendizaje se debe evaluar también por escrito, o bien en pruebas parciales breves después de cada práctica (al final de la clase o al principio de la clase siguiente con una duración aproximada de quince o veinte minutos), o bien en una prueba al final del curso, con preguntas relativas a las distintas actividades complementarias realizadas a lo largo del mismo. De esta manera la evaluación de la parte práctica es continua y el trabajo autónomo, mientras que el esfuerzo individual no queda opacado en una calificación únicamente por grupos.

Llegados a este punto, todo parece indicar que el instrumento que aporta coherencia y da sentido a todo nuestro conjunto de prácticas es, como ya hemos dicho, la guía de lectura. ¿En qué consisten estas guías? ¿Por qué elaborar un instrumento de trabajo de estas características para las actividades prácticas?

\section{2.- LA LECTURA INTERMEDIADA: LAS GUÚAS DE LECTURA}

La idea esencial que subyace a la elaboración de este instrumento de trabajo es la necesidad de mejorar el ejercicio de la lectura comprensiva de textos académicos por parte de los estudiantes. Esto, obviamente, ha de redundar a corto y medio plazo en la mejora del proceso de enseñanza y aprendizaje, no sólo en las materias que nos ocupan sino del conjunto del Grado en Relaciones Laborales y Recursos Humanos. Pero las guías constituyen, ante todo, un intento de dar respuesta a una carencia en la comprensión lectora detectada a partir de nuestra experiencia docente y para explicarla debemos contextualizar adecuadamente el grupo humano para el que se construye esta herramienta.

Para empezar, la población estudiantil que ingresa al Grado de Relaciones Laborales y Recursos Humanos conforma cinco grupos muy numerosos (en torno a los setenta y hasta ochenta alumnos matriculados por cada grupo) en la asignatura del primer curso, "Historia de las Relaciones Laborales", y un solo grupo mucho más pequeño para la asignatura optativa de segundo curso, "Historia Económica del Trabajo". Los alumnos provienen, en general, de Institutos de Educación Secundaria de los pueblos y ciudades alrededor de Sevilla y en menor medida de la capital; la mayoría de los estudiantes ronda los 18-20 años en los grupos de la mañana, mientras que encontramos una mayor diversidad de edades y motivaciones en los grupos de la tarde, donde se concentra claramente la población estudiantil mayor de 25 años, que compagina usualmente los estudios con un empleo remunerado.

Su formación académica previa es heterogénea. Salvo contadas excepciones, los alumnos no se han enfrentado previamente a la lectura de un texto académico y muy pocos demuestran verdadera soltura en la redacción de un ensayo o síntesis o en la exposición de un tema frente al público en el aula. Las habilidades que se 
suponen, pues, inherentes al horizonte universitario en el que se sumergen — saber reducir un texto cualquiera a sus ideas esquemáticas o estructurarlo posteriormente con base en un ordenamiento lógico y coherente- se hallan asimismo ausentes o muy débilmente asentadas en estos primeros cursos.

La guía consta de una serie de preguntas cortas —entre veinte y treinta normalmente- que se resuelve íntegra y ordenadamente a partir de la lectura (o proyección fílmica) a la que se refiere;' es decir, todas las preguntas siguen un orden consecutivo, no obligan al alumno a volver sobre sus pasos o adelantarse en el texto, ni a establecer inferencias a partir de información externa al mismo. La guía, en este sentido, supone una intermediación crítica entre el lector y el texto académico, en tanto efectúa sucesivamente las preguntas que el texto va contestando. Las preguntas están cuidadosamente elegidas y planteadas, son directas, formuladas con arreglo a una redacción y vocabulario similares a los utilizados en el texto, cuyo fin es desintegrarlo en sus ideas cardinales. ${ }^{10}$ Cada pregunta contribuye a fijar la atención en los conceptos básicos de la lectura y aclara su estructura interna. Se intenta, pues, que los alumnos lleguen, por una vía más sencilla, a los contenidos esenciales de los textos y tratamos de evitar que su atención se disperse hacia aspectos secundarios, anecdóticos o incluso inventados.

Desde el punto de vista de las competencias transversales que las asignaturas de Historia en este Grado deben cumplir (citadas más arriba), el proceso de lectura comprensiva que los alumnos efectúan a través de la guía se corresponde con el primer punto de dichas competencias, la capacidad de análisis y síntesis. En efecto, ese desmenuzamiento de los textos al que lleva esta herramienta refleja el proceso de análisis de la información, esto es, la operación de pensamiento que supone la división de situaciones o tareas complejas en otras más sencillas con miras a facilitar la comprensión y profundización de los conocimientos extraídos de un texto. Pero además, en las últimas preguntas de las guías (Anexo I) siempre solicitamos, antes, las cinco o seis ideas más importantes del texto de modo que, a partir de ellas, se elabore, después, una síntesis del mismo. Y con este último ejercicio, que probablemente supone el mismo tiempo y esfuerzo que lleva resolver el resto de preguntas, los estudiantes realizan la labor complementaria al análisis, la síntesis, el proceso mediante el cual se integran las partes, las propiedades y las relaciones de un conjunto delimitado para formar un todo significativo, una visión global, una estructura coherente.

Respecto al resto de competencias transversales, si la lectura de un texto puede aportar los conocimientos generales básicos contemplados en los contenidos teóricos

9. Véase Anexo I.

10. No se trata, pues, de preguntas "con trampa", ni con planteamientos que pongan en guardia a los alumnos o creen confusión por el lenguaje o la forma de preguntar. Su sencillez es la clave principal de uso: dan las pautas para que ellos descubran con este apoyo las partes más importantes del texto. 
de las asignaturas, las guías constituyen los instrumentos que permiten llegar hasta ellos. Por otro lado, mientras que el trabajo en casa con las guías desarrolla la habilidad para recuperar y analizar información, el trabajo en grupos en el aula refuerza la capacidad crítica y autocrítica de los alumnos. Creemos, en definitiva, que esta guía constituye un refuerzo tanto en la capacidad de aprender como en el estímulo para hacerlo, pues no deja solos a los estudiantes ante textos a los que no se han enfrentado en los anteriores ciclos educativos y para los que, en muchos casos, no suelen tener instrumentos didácticos propios con los que abordarlos.

\section{3.- $\quad$ EVALUACIÓN DEL USO DE LAS GUÍAS DE LECTURA ENTRE LOS ALUMNOS DE LA FACULTAD DE CIENCIAS DEL TRABAJO}

\section{1.- LA HERRAMIENTA DE ANÁLISIS}

Con el objeto de evaluar el impacto de la guía de lectura como instrumento docente, se construyó un cuestionario tipo en el que quedase reflejada la percepción de los alumnos sobre la mecánica y desarrollo de las actividades prácticas realizadas durante el curso. La herramienta fue diseñada teniendo en cuenta dos aspectos básicos.

En primer lugar, los ámbitos de control debían quedar claramente definidos, optándose finalmente por la siguiente distribución: facilidad (sencillez en el aprendizaje), adecuación al programa de la asignatura, aprovechamiento dentro del ciclo de estudio, exigencia de trabajo previo de preparación del ejercicio y eficacia de la sesión de puesta en común en el seminario. Por último, y a modo de pregunta de control, se incluyó un último criterio de valoración global.

En segundo lugar, con el objeto de evaluar los resultados, se introdujo un criterio de jerarquización entre prácticas de acuerdo al mayor o menor grado de conformidad con la variable propuesta. La jerarquización plantea problemas y restricciones al análisis al trabajar con variables discretas, pero así se evita la sobrecarga estadística de los valores intermedios. ${ }^{11}$

Así pues, todas las encuestas han seguido el mismo cuestionario y el mismo patrón de valoración, pero tanto el número como la naturaleza de los ejercicios prácticos propuestos por cada profesor han sido diferentes. Ante la diferencia en el número de ejercicios prácticos propuestos por cada profesor y, en consecuencia, las distintas desviaciones en los valores, se ha optado por agrupar los datos en tres intervalos no homogéneos: bajo, medio y alto. Es decir, para reducir la deformación estadística producida por los valores extremos correspondientes a los grupos que mayor número de prácticas han realizado (que, además, constituyen la muestra más numerosa), se ha

11. Véase una ficha tipo en el Anexo II. 
incrementado el peso estadístico de los valores centrales, aumentando así la dimensión del intervalo medio para adecuarlo al tamaño del resto de grupos.

Según su tipología, los ejercicios prácticos propuestos han sido agrupados en cuatro categorías diferentes: texto de investigación, fragmento de manual u otro material docente de lectura, vídeo documental y sesión de conferencia académica. Cada una de estas categorías podía utilizar o no como herramienta la guía de lectura. De las ocho categorías posibles, en el caso del actual estudio se reducen a seis, al no proponerse todas las posibilidades.

En razón de la tipología de los ejercicios propuestos, las muestras se distribuyen de la siguiente manera:

CUADRO 1. Tipología de las Prácticas REalizadas y eValuadas

\begin{tabular}{|c|c|c|c|c|}
\hline TIPOLOGÍA & $\begin{array}{c}\text { Texto } \\
\text { Investigación }\end{array}$ & $\begin{array}{c}\text { Texto manual/ } \\
\text { docente }\end{array}$ & Vídeo documental & $\begin{array}{c}\text { Conferencia } \\
\text { académica }\end{array}$ \\
\hline Con guía de lectura & 15 & 2 & 2 & 6 \\
\hline Sin guía de lectura & 3 & 0 & 2 & 0 \\
\hline
\end{tabular}

Como puede observarse en el cuadro 1, existe una gran sobrecarga estadística en el campo de los textos de investigación: esto se debe a la existencia de una serie de ejercicios consensuados por el equipo docente, y propuestos en todos los grupos. Más allá del desequilibrio que pueda plantear, aporta al estudio un punto de control, al ser el elemento que cuenta con la mayor frecuencia acumulada de respuestas.

\section{2.- UNIVERSO}

El universo considerado es el de los alumnos de la asignaturas de $\mathrm{H}^{\mathrm{a}}$ de las RR.LL. de la Facultad de CC. del Trabajo de Sevilla. Esto compone un total de 523 alumnos. Nuestra muestra está formada por 222 alumnos, que corresponden a un 42 por 100 de los matriculados. Este porcentaje es representativo de los que han seguido el curso con asiduidad y hay que considerar que la encuesta se realizó en los días finales del curso, por lo que la evaluación de dos de los ámbitos estudiados (la adecuación de las prácticas al programa y su nivel de aprovechamiento) se efectuó en el momento en que la percepción del alumno se encuentra más activa.

\section{3.- Evaluación de Resultados}

Los datos han sido desagregados en dos gráficos diferentes (gráficos 1 y 2), atendiendo al carácter de las prácticas evaluadas: aquellas basadas en la lectura de un texto y aquellas con otro soporte, vídeo o conferencia. 
Entre las correspondientes a lecturas, se ha de advertir que las prácticas se realizaron cronológicamente en el mismo orden con que aparecen en el gráfico 1. Una primera característica que se observa es el alto peso estadístico de las calificaciones medias en el ámbito de los textos de investigación apoyados por guía de lectura. Esta particularidad es consecuencia, fundamentalmente, de la ya mencionada variación introducida en el rango de los intervalos de jerarquización, con el objeto de evitar la deformación de las calificaciones extremas (baja y alta); y del orden cronológico de la realización de las pruebas. La alta concentración de este tipo de ejercicios en el comienzo del curso, un 70 por 100 del total de las dos primeras sesiones de prácticas, los convierte en la primera toma de contacto del alumno con esta dimensión de la asignatura, pero lejanos en el momento en el que se realiza la encuesta, es decir, al término del curso y en pleno proceso de preparación de los exámenes.

Gráfico 1. Grado de CONFOrmidad de los alumnos a las prácticas realizadas a partir De lecturas (alto, MEdio y Bajo, EN PORCENTAje)

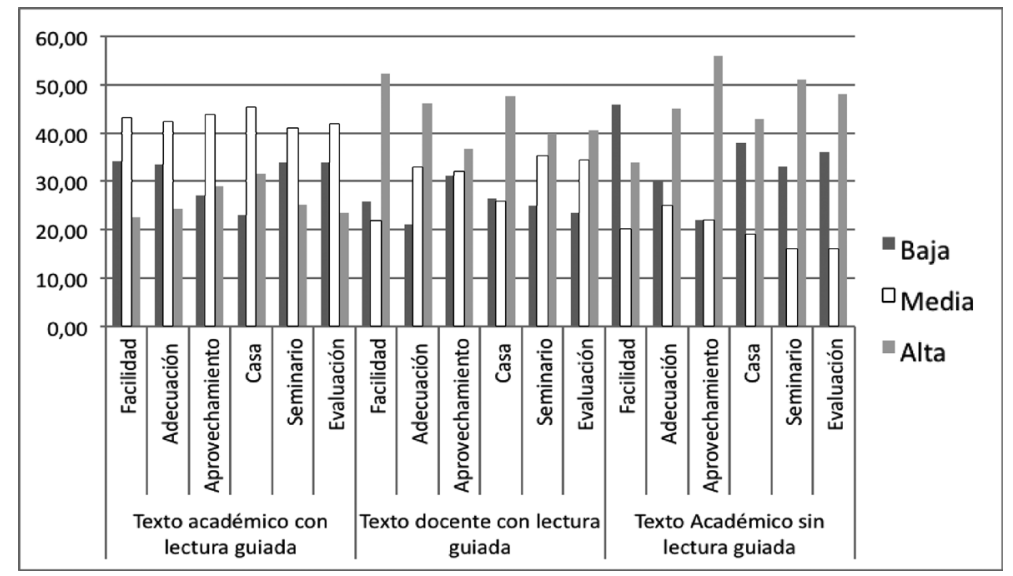

Fuente: Encuestas a 222 alumnos de Historia de las Relaciones Laborales. Grado de Ciencias del Trabajo, Universidad de Sevilla. Curso 2011-2012. Primer Cuatrimestre.

Globalmente, si distinguimos entre las prácticas apoyadas o no con guía de lectura, puede observarse cómo el uso de la guía hace aumentar notablemente los valores medios respecto a los valores extremos. A nuestro juicio, ésta constituye la inferencia principal del ejercicio: la guía de lectura acorta la distancia entre el bagaje cultural del alumno y el texto, de manera que su percepción se normaliza.

La introducción de la guía de lectura aporta peso a los valores medios respecto al grado de facilidad de una lectura, incluso en los textos académicos, aunque los textos procedentes de manuales son siempre percibidos como más accesibles, más aún si se 
dispone de una guía de lectura. En el caso de la percepción sobre el aprovechamiento y la adecuación de las prácticas sin guía de lectura, llama la atención los altos valores de conformidad de los alumnos. Pese a su mayor dificultad relativa (valores bajos y medios de facilidad), los alumnos realizaron más tardíamente esta lectura, además de ser capaces de discernir que el contenido de los textos era de mayor utilidad o adecuación para su aprendizaje. Atendiendo al volumen de trabajo previo exigido por las prácticas, la ausencia de guía de lectura fomenta la paradoja de aumentar los valores extremos. Entendemos que la ausencia de guía de lectura como instrumento de ayuda obliga a un régimen de trabajo de alta intensidad. Algunos alumnos agradecerán el librarse de una herramienta que les obliga a prestar mayor atención, mientras que otros la echan en falta. La introducción de la guía mitiga indudablemente dicho esfuerzo, reduciendo los valores extremos.

Un aspecto en el que aparecen grandes divergencias es en el ámbito del aprovechamiento. En este caso, volviendo a argumentos ya mencionados, se entiende que su variabilidad denota más el grado de ajuste de la lectura, y de la propia práctica, con la dinámica del resto del curso -incluidas las clases magistrales-que cualquier otra circunstancia.

Al pasar al segundo módulo de texto, el material docente o procedente de manual, lo primero a tener en cuenta es el cambio rotundo respecto a los ejercicios basados en textos de investigación. En efecto, el texto docente, en este caso dotado además de guía de lectura, se presenta mucho más accesible al alumno, con un alto grado de facilidad. En general, su adecuación al temario se percibe como mayor y, aunque plantea una carga de trabajo notable, es en el aprovechamiento global y en la sesión de seminario donde las percepciones se hallan más divididas.

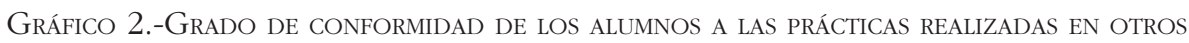
SOPORTES (ALTO, MEDIO Y BAJO, EN PORCENTAJE)

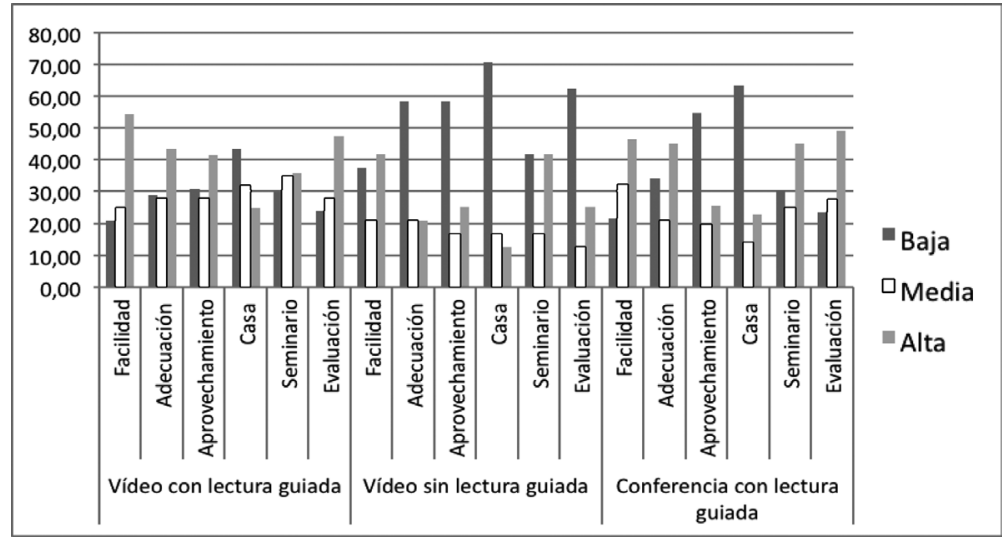

FUENTE: Encuestas a 222 alumnos de Historia de las Relaciones Laborales. Grado de Ciencias del Trabajo, Universidad de Sevilla. Curso 2011-2012. Primer Cuatrimestre. 
Al observar la segunda agregación de datos (véase gráfico 2), las distribuciones muestran un aspecto más claro que en las correspondientes a las lecturas. Comenzando por los ejercicios basados en la proyección de vídeo documental, las diferencias entre el uso o no de la guía de lectura como instrumento de apoyo se hacen patentes a primera vista.

En primer lugar, y atendiendo a los índices de facilidad, la percepción de media/ elevada es compartida por casi el 80\% de los alumnos al contar con guía de lectura, proporción que se reduce a un 60\% al retirar la herramienta. En segundo lugar, la dificultad para ubicar la adecuación del ejercicio al programa asciende de un 28 por 100 a un 58 por 100 en caso de no contar con la guía. De acuerdo con lo anterior, la percepción del grado de aprovechamiento sigue pautas y proporciones similares.

En la valoración de la sesión de seminario, se plantea una interesante divergencia: mientras que aquellos alumnos que han contado con la guía como apoyo se distribuyen de forma uniforme, en el caso de los demás son los valores extremos los que adquieren mayor peso estadístico, planteándose unos resultados muy asimétricos: el 42 por 100 percibe el seminario como poco útil frente a otro 42 por 100 que lo considera muy productivo. En último lugar, respecto a la valoración global, las diferencias entre uno y otro tipo se muestran como consecuencia de lo anterior: a una valoración media/alta dada por más del 75 por 100 de los alumnos que utilizaron guía de lectura, se opone el bajo nivel percibido por más del 60 por 100 de los que no contaron con esta.

Por último, al analizar los datos referidos a las prácticas basadas en conferencias académicas, se observa cómo las distribuciones de datos se mantienen al alza en los apartados de facilidad, adecuación y en la sesión de seminario.

En su conjunto, las valoraciones de los alumnos de Historia de las Relaciones Laborales en Ciencias del Trabajo muestran que, a su llegada a la Universidad, están tan familiarizados con la lectura de manuales como con el aprendizaje a partir de otros soportes, como son los vídeos y las conferencias. En contraste, la lectura de textos académicos — artículos o capítulos de libros - constituye un reto para ellos que, sin embargo, las guías de lectura contribuyen a superar satisfactoriamente. Esto no significa que los alumnos perciban los textos y los vídeos más sencillos como los más provechosos para su aprendizaje, o los más adecuados al programa. Antes bien, pese a la dificultad de los textos académicos, los alumnos se sobreponen a ella, siempre y cuando su lectura revista interés. A nuestro juicio, estos resultados nos animan a no renunciar a la lectura de textos académicos con los alumnos de primero, aunque sigamos combinándola con vídeos y conferencias, a seleccionar adecuadamente las lecturas y a utilizar siempre que sea posible una guía que establezca ese necesario puente entre su capacidad lectora al comienzo del curso y la necesaria para la total comprensión de un texto académico. 


\section{4.- $\quad$ CONCLUSIONES}

Como la cultura lectora que impera en la educación secundaria es muy diferente a la que se exige en la educación universitaria, el paso de la cultura académica característica de la educación secundaria a la nueva cultura académica propia de la educación superior precisa de un proceso de "alfabetización académica". Pese a que nosotros, profesores, solemos pensar que los estudiantes desarrollarán las nuevas habilidades lectoras requeridas a su llegada a la Universidad de manera espontánea e inmediata, lo cierto es que muchos estudiantes naufragan en esas aguas.

La reforma educativa de la enseñanza superior en la que estamos inmersos nos exige repensar totalmente el proceso de enseñanza aprendizaje. La transmisión de conocimientos de forma unidireccional está dando paso a la adquisición pro-activa por parte de los alumnos de un amplio abanico de competencias genéricas (instrumentales, personales y sistémicas) y específicas (disciplinares, profesionales y académicas).

El propósito de la guía de lectura en el marco de las clases prácticas de las asignaturas de Relaciones Laborales en la Facultad de Ciencias del Trabajo es aplicar una estrategia didáctico-metodológica donde el alumno sea un elemento activo en el proceso de enseñanza aprendizaje, creando las condiciones adecuadas para su aprendizaje autónomo y continuo y procurando dar los primeros pasos en la "alfabetización académica del alumno".

Para nosotros, profesores, la experiencia de este curso académico nos lleva a considerar que la aplicación de esta estrategia didáctica ha logrado crear las condiciones adecuadas para que el aprendizaje sea autónomo, reforzando su capacidad de análisis y síntesis, su motivación y su capacidad para leer, comprender y aprehender. Para los alumnos, según reflejan los resultados de las encuestas efectuadas, el acercamiento a los textos académicos --artículos o capítulos de libros-- constituye un desafío que las guías de lectura ayudan a vencer adecuadamente. Sus respuestas nos animan a fomentar la "alfabetización académica" como competencia instrumental para los alumnos de primer curso. Propondremos la lectura de textos académicos, allanados mediante las guías de lectura, aunque no renunciemos a los vídeos y las conferencias. Sabemos que debemos mejorar en la selección de las lecturas, adecuarlas mejor al devenir del curso y al aprendizaje gradual por parte de los estudiantes, de manera que el conjunto de las prácticas provean de ese puente necesario entre su capacidad lectora al comienzo del curso y la que se requiere para la total comprensión de un texto académico.

\section{5.- BIBLIOGRAFÍA}

ARENAS POSADAS, Carlos, FLORENCIO PUNTAS, Antonio y Jerònia PONS PONS, (coords.) (2001): Trabajo y relaciones laborales en la España contemporánea, Sevilla, Mergablum. 
ARENAS POSADAS, Carlos, FLORENCIO PUNTAS, Antonio y José Ignacio MARTÍNEZ RUIZ (coords.) (1999): Mercados y organización del trabajo en España (siglos XIX y XX), Sevilla, Atril.

BERNAL RODRÍGUEZ, Antonio Miguel, COLLANTES DE TERÁN SÁNCHEZ, Antonio y GARCÍA-BAQUERO GONZÁLEZ, Antonio (coords.) (2008): Sevilla, de los gremios a la industrialización, Sevilla, Universidad de Sevilla.

CARLINO, Paula (2003 a), "Alfabetización académica: un cambio necesario, algunas alternativas posibles", en Educere. Revista Venezolana de Educación, vol. 6, $\mathrm{n}^{\circ}$ 20, pp. 409-420. En: http://www.saber.ula.ve/bitstream/123456789/19736/1/articulo7.pdf [Consultado el 3 de mayo de 2012].

CARLINO, Paula (2003 b): "Leer textos complejos al comienzo de la educación superior. Tres situaciones didácticas para afrontar el dilema", en Textos. Didáctica de la Lengua y la Literatura, n 33, abril-mayo, pp. 43-51.

CARLINO, Paula (2006): Escribir, leer y aprender en la universidad. Una introducción a la alfabetización académica, Fondo de Cultura Económica, México, $2^{\text {a }}$ reimp.

CASSANY, Daniel (2006): Taller de textos. Leer, escribir y comentar en el aula, Barcelona, Paidós.

ESTIENNE, Viviana M. (2004): "Enseñar a leer en la universidad. Una responsabilidad compartida”, en I Congreso Internacional Educación, Lenguaje y Sociedad. Tensiones educativas en América Latina, Universidad Nacional de La Pampa, del 1 al 3 de julio de 2004. En: http://www.fchst.unlpam.edu.ar/iciels/127. pdf [Consultado el 3 de mayo de 2012].

FLORES, María Luz y NATALE, Lucía (2004): “Cómo ayudar a los docentes universitarios a implementar la enseñanza de la lectura y la escritura?", en I Congreso Internacional Educación, Lenguaje y Sociedad. Tensiones educativas en América Latina, Universidad Nacional de La Pampa, del 1 al 3 de julio de 2004. En: http://www.fchst.unlpam.edu.ar/iciels/130.pdf [Consultado el 3 de mayo de 2012].

GALÁN GARCÍA, Agustín (2011): "El proceso de convergencia desde el Grado de Relaciones Laborales y Recursos Humanos: una ojeada al panorama nacional", en MARTÍN LÓPEZ, Milagro y Adela ROLDÁN MÁRQUEZ (coords.), EEES y cambios en las metodologias docentes. Reflexiones y experiencias en su aplicación a las ciencias del Trabajo, Valencia, Tirant lo Blanch, pp. 15-45. 
GALÁN GARCíA, Agustín y Domingo A. MANZANARES MARTínEZ (2010): "El área de Historia e Instituciones Económicas en el Grado de Relaciones Laborales y Recursos Humanos: orientaciones, contenidos y competencias", en Actas del IX Encuentro de Didáctica de la Historia Económica, Toledo, del 24 al 25 de junio de 2010. Disponible en: http://www.uclm.es/ area/Hinstituciones/IXEncuentro HE Toledo/trabajos.htm [Consultado el 24 de abril de 2012].

GALÁN GARCÍA, Agustín y Ma Sol HERRAIZ MARTÍN (2005): "La enseñanza de las Relaciones Laborales en España", en Trabajo. Revista de la Asociación Estatal de Centros Universitarios de Relaciones Laborales y Ciencias del Trabajo, 15, enero-junio, Servicio de Publicaciones de la Universidad de Huelva, pp. 17-31.

GEISLER, Cheryl (1994): Academic Literacy and the Nature of Expertise. Writing and Reading in Academic Pbilosophy, Hillsdale, Lawrence Erlbaum Associates.

GONZÁleZ ARCE, José Damián, MANZANARES MARTíneZ, Domingo Antonio, MARTÍNEZ SOTO, Ángel Pascual y Josep María RAMÓN MUÑOZ (2008): "Aportaciones de la Historia Económica al Grado de Ciencias Laborales y Recursos Humanos”, en PÉREZ HERNÁNDEZ, Concepción (coord.), Actas del VIII Encuentro de Didáctica de la Historia Económica, La Laguna, (Tenerife), del 20-21 de septiembre de 2007, pp. 273-288.

HYLAND, Ken (2000): Disciplinary Discourses: Social Interactions in Academic Writing, London, Longman.

JOHnS, Ann M. (1997): Text, Role and Context. Developing Academic Literacies, Cambridge, Cambridge University Press.

LERMA MONTERO, Ignasi (coord.) (2005): Libro Blanco del Título de Grado en Ciencias Laborales y Recursos Humanos, Agencia Nacional de Evaluación de la Calidad y la Acreditación (ANECA). Disponible en: http://www.aneca.es/activin/docs/libroblanco rrhh def.pdf [Consultado el 24 de abril de 2012].

NARVÁEZ CARDONA, Elizabeth y CADENA CASTILLA, Sonia (comps.) (2008): Los desafios de la lectura y la escritura en la educación superior. Caminos posibles, Cali, Universidad Autónoma de Occidente.

NARVAJA DE ARNOUX, Elvira, DI STEFANO, Mariana y PEREIRA, Cecilia (2002): La lectura y la escritura en la universidad, Buenos Aires, Eudeba.

THOMPSON, E. P. (1979): "Tiempo, disciplina de trabajo y capitalismo industrial", en Tradición, revuelta y consciencia de clase, Barcelona, Crítica, pp. 258-290. 


\section{ANEXOS}

Anexo I: Guía de lectura del texto de E. P. Thompson.

Facultad de Ciencias del Trabajo

Grado en Relaciones Laborales y Recursos Humanos

Asignatura: Historia Económica del Trabajo

GUÍA DE LECTURA

\section{Texto:}

THOMPSON, E. P. (1979), "Tiempo, disciplina de trabajo y capitalismo industrial", en Tradición, revuelta y consciencia de clase, Barcelona, Crítica, pp. 258-290.

\section{Preguntas:}

1.- ¿De qué manera se entendió el elemento del tiempo mientras la industria manufacturera se mantuvo en una escala doméstica? ¿Por qué?

2.- ¿Cuál es, entonces, la principal característica de las normas de trabajo preindustriales y por qué?

3.- ¿Por qué dice el autor que en tipo de trabajos preindustriales no podía sobrevivir una planificación del tiempo precisa?

4.- ¿Cómo era la norma de trabajo propia de la etapa preindustrial y cómo llegó a ser considerada?

5.- ¿Qué formas de dicha irregularidad del tiempo de trabajo eran las más extendidas entre los trabajadores antes del advenimiento de la fábrica? ¿Podría describir las más importantes?

6.- En el relato del Viejo Alfarero ¿Cómo se explica desde el punto de vista de los hábitos de trabajo la pervivencia de San Lunes?

7.- ¿A qué factor atribuía el escritor de este relato (un predicador metodista liberal-radical) esta costumbre "deplorable" del San Lunes?

8.- Según el autor ¿Hasta qué punto era extensible esta situación a los trabajadores rurales? ¿Podían tener los jornaleros agrícolas las mismas costumbres irregulares?

9.- ¿Cómo influyeron el cerramiento de los campos y el progreso agrícola en la disciplina del tiempo de la mano de obra?

10.- ¿En qué consistió el debate sobre las diferentes formas de pago del trabajo a fines del siglo XVIII y durante el siglo siguiente?

11.- ¿Cómo se caracteriza el trabajo femenino rural en este texto? ¿Con qué argumentos extrapola el autor la situación de la mujer entonces con la del mundo contemporáneo? ¿Están de acuerdo con esta opinión?

12.- ¿A qué elemento le atribuye este autor una gran importancia en el tiempo de transición hacia la industrialización en países como Inglaterra o Francia? ¿Por qué? ¿Cómo caracteriza Thompson el tipo de transición del que está hablando? 
13.- ¿Por qué fue tan prolongada y plagada de conflictos la transición en Gran Bretaña?

14.- ¿Por qué fueron tan severas las doctrinas mercantilistas en cuanto a mantener bajos los salarios?

15.- ¿Qué aspectos de la disciplina del tiempo fueron abordados por el Law Book of the Crowley Iron Works? ¿Qué figuras creaba este código y para qué?

16.- A falta de maquinaria ¿Qué medidas disciplinarias fueron impuestas por Josiah Wedwood en los talleres de alfarería que dirigía?

17.- ¿Cómo se trató de imponer el ahorro del tiempo en los distritos de manufactura domésticos tal y como aparece explicado en el folleto del reverendo Clayton Friendly Advice to the Poor? ¿Consiguió sus propósitos?

18.- ¿A qué otra institución no industrial apela el reverendo Clayton en sus Friendly Advice? ¿Por qué? ¿Cómo consideran otros autores contemporáneos a la educación?

19.- ¿Cómo se desarrolló la oposición a todo este tipo de normas contra los hábitos de trabajo tradicionales?

20.- En oficios excepcionales y con un mercado de mano de obra favorable ¿Cómo se desarrolló la disciplina del tiempo?

21.- ¿Cómo se desarrolló la contienda sobre las horas de trabajo en las industrias? ¿Por qué?

22.- ¿Cómo expresa el autor la idea de que los obreros finalmente aceptaron las nuevas normas de disciplina del tiempo en las fábricas?

23.- ¿De qué manera opina Thompson que fue interiorizada y asumida esta nueva disciplina del tiempo por los artesanos y obreros? ¿Qué dos factores se citan en la apelación de los moralistas al aprecio del tiempo?

24.- ¿Cómo desarrollaron los predicadores, metodistas y los evangelistas, el tema del gobierno del tiempo?

25.- ¿Cómo expresó Benjamin Franklin su punto de vista sobre el tiempo?

26.- ¿De qué modos se impuso la nueva disciplina de trabajo y con qué ritmo y dónde fundamentalmente?

27.- ¿En qué se especificaba la crítica de los moralistas del XIX (la llamada "época victoriana en Inglaterra) hacia el "ocio de las masas"?

28.- El autor supone que la nueva disciplina se interiorizó realmente y fue exitosa ¿A través de qué canales propagandísticos y qué incentivos fue posible este cambio?

29.- ¿Qué valores expresan este cambio e hicieron distinguirse a los obreros ingleses del XIX con otros obreros de otros países?

30.- El autor establece un paralelismo entre los trabajadores ingleses "pobres" del XVIII y los peones mexicanos de principios del siglo XX ¿Qué valores supone comunes en ambos ejemplos? 
31.- ¿Cómo ejemplifica Thompson actualmente (en los países en vías de desarrollo) las formas en las que los trabajadores se han ido adaptando a diferentes disciplinas del tiempo?

32.- A partir de la caracterización de las sociedades industriales como aquellas que administran el tiempo y distinguen "trabajo" de "vida": ¿Qué crítica formula el autor que subyace más allá de un cambio hacia un "mejor nivel de vida"?

33.- Pregunta general final: Qué tres o cuatro aspectos/ideas les parece a ustedes que son los ejes vertebradores de este texto?

34.- Haga un resumen breve del texto

Anexo II: Ficha tipo de evaluación.

Facultad de Ciencias del Trabajo

Asignatura: Historia Económica del Trabajo

Curso 2011-2012

Evaluación de las Actividades ComplementaRias

1 de febrero de 2012

\section{INSTRUCCIONES}

Esta evaluación es anónima. No es necesario que escribáis vuestro nombre y apellidos.

Os rogamos que leáis estas instrucciones con atención:

La evaluación consiste en ORDENAR de 1 a 7 cada columna donde:

(1) es el minimo

(7) es el máximo

Un EJEMPLO con la última columna de Evaluación Final:

\begin{tabular}{|c|c|}
\hline Prácticas realizadas & Evaluación final \\
\hline Thompson & 5 \\
\hline Montgomery & 4 \\
\hline Toharia & 1 \\
\hline Tolliday & 3 \\
\hline Jacoby & 2 \\
\hline Galán & 7 \\
\hline Arenas & 6 \\
\hline
\end{tabular}


Cada COLUMNA corresponde a una pregunta distinta:

En la primera columna se pide que ordenes las siete prácticas entre la qué te pareció más fácil (7) y la más complicada (1).

En la segunda debes ordenar desde la que más te sirvió, la que más aprovechaste, con la que aprendiste más, hasta la que menos provechosa te resultó.

En la tercera debes ordenar desde la práctica que te pareció más relacionada con el temario a la que menos.

En la cuarta columna se pide que ordenes de mayor a menor el esfuerzo y el trabajo realizado en casa con cada una de las prácticas.

En la quinta columna ordena las sesiones prácticas en clase, desde las que más interesantes y más provechosas te resultaron hasta las que menos.

En la última columna pedimos que valores en su conjunto cada práctica; un 7 para la que te pareció la mejor de todas, un 1 para la que menos te gustó.

\section{EVALUACIÓN}

\begin{tabular}{|c|c|c|c|c|c|c|c|}
\hline & PRÁCTICAS & $\begin{array}{l}\text { FACILIDAD } \\
\text { DE LA } \\
\text { PRÁCTICA }\end{array}$ & $\begin{array}{l}\text { GRADO DE } \\
\text { APROVECHA. }\end{array}$ & $\begin{array}{l}\text { ADECUACIÓN } \\
\text { AL TEMARIO }\end{array}$ & $\begin{array}{l}\text { TRABAJO EN } \\
\text { CASA }\end{array}$ & $\begin{array}{l}\text { PRÁCTICAS } \\
\text { EN CLASE }\end{array}$ & $\begin{array}{l}\text { EVALUACIÓN } \\
\text { FINAL }\end{array}$ \\
\hline 1 & $\begin{array}{l}\text { Thompson: } \\
\text { tiempo y } \\
\text { disciplina... }\end{array}$ & & & & & & \\
\hline 2 & $\begin{array}{l}\text { Montgomery: } \\
\text { control obrero... }\end{array}$ & & & & & & \\
\hline 3 & $\begin{array}{l}\text { Toharia: } \\
\text { extracción de } \\
\text { trabajo... }\end{array}$ & & & & & & \\
\hline 4 & $\begin{array}{l}\text { Tolliday: } \\
\text { Difusión del } \\
\text { fordismo }\end{array}$ & & & & & & \\
\hline 5 & $\begin{array}{l}\text { Jacoby: } \\
\text { mercados } \\
\text { internos de } \\
\text { trabajo... }\end{array}$ & & & & & & \\
\hline 6 & $\begin{array}{l}\text { A. Galán: } \\
\text { accidentes de } \\
\text { trabajo... }\end{array}$ & & & & & & \\
\hline 7 & $\begin{array}{c}\text { C. Arenas: crisis } \\
\text { y neoliberalismo } \\
\text { actual... }\end{array}$ & & & & & & \\
\hline
\end{tabular}


